\title{
Effect of Nuclear Motion on the Energy Eigenvalues in Muonic Atoms*
}

\author{
B. Fricke $\dagger$ \\ Department of Materials Science, Northwestern University, Evanston, Mlinois 60201 \\ (Received 24 July 1972)
}

\begin{abstract}
I have investigated the effect of the nuclear motion on the energy eigenvalues in muonic atoms. In addition to the usually used reduced-mass correction, I have calculated the relativistic influences including the magnetic and retardation interaction between the nucleus and the muon for the inner orbitals of muonic atoms.
\end{abstract}

A muonic atom, like the hydrogen atom, is in principle the simplest of all possible atoms. It is necessary, however, to add a number of corrections to the hydrogenic values to get accurate theoretical values to compare with the observed $\mathrm{x}$-ray energies. The largest of all corrections for inner orbitals comes from the influence of the extended nucleus in heavy muonic atoms. It reduces the $l s$ binding energies by more than $50 \%$ from the value for a point nucleus. This provides an excellent possibility to determine the nuclear charge distribution. Many papers have dealt with this subject, ${ }^{1}$ and a great amount of information on the nuclear radii, etc. has been gathered. The other corrections which have been discussed in some detail are the vacuum polarization, ${ }^{2-4}$ the Lamb shift, ${ }^{5}$ the nuclear polarization, ${ }^{6}$ and the electron shielding. ${ }^{7}$

In the nonrelativistic therry, the effect of the nuclear motion is fully taken into account by using the reduced mass $\mu=m M /(m+M)$ of the system instead of the rest mass $m$ of the muon, where $M$ is the nuclear mass. In its simplest form, this correction is given by the energy eigenvalue multiplied by $m / M$. But even in the nonrelativistic theory this is only valid as long as the nucleus is a point charge, because the virial theorem does not hold true in the region inside the nucleus. In addition to that, the reduced-mass effect is not fully taken into account in the relativistic theory when the reduced mass is used instead of the rest mass. This so-called relativistic reduced-mass correction was first calculated by Bechert and Meixner ${ }^{8}$ and later by Breit and Brown ${ }^{9}$ mainly to show that this effect is not significant for the fine structure or the Lamb shift of electronic levels in hydrogen. In view of the high accuracy of recent measurements of the transition energies in the muonic atoms this statement is no longer true. Therefore some authors ${ }^{5,1}$ have used a correction formula for the relativistic reduced-mass correction,

$$
-(Z \alpha / 2 n)(m / M)\left|W-m c^{2}\right|^{2}
$$

where $W$ is the total energy of the bound muon. But this formula is only applicable to states where the relativistic effects as well as the influence of the extended nucleus are very small. Breit and Brown examined the whole question from a general point of view using a Hamiltonian applicable to two charged particles and accounting for effects of the order of $v^{2} / c^{2}$, where $v$ is the velocity of the bound particle. The solution is carried out in terms of an eight-component approximation of the sixteen-component wave function. They show that the only important contributions for the effect of the nuclear motion, and this is also true for all levels of all possible muonic atoms, can be expressed in terms of the sum of the expectation values of the quantities

$$
E_{1}=\left\langle\frac{p^{2}}{2 M}\right\rangle+\frac{Z e^{2}}{4 M c}\left\langle\left|\frac{\overrightarrow{\mathrm{p}} \cdot \vec{\alpha}}{r}+\frac{\vec{\alpha} \cdot \overrightarrow{\mathrm{p}}}{r}+\frac{(\overrightarrow{\mathrm{p}} \cdot r)(\vec{\alpha} \cdot r)}{r^{3}}+\frac{(\vec{\alpha} \cdot r)(\overrightarrow{\mathrm{r}} \cdot \overrightarrow{\mathrm{p}})}{r^{3}}\right|\right\rangle
$$

where $|\vec{p}|$ is the momentum of the nucleus and the muon, $\vec{\alpha}$ the $4 \times 4$-component Dirac matrices, and $\vec{r}$ the distance between the nucleus and the bound particle. The first part of Eq. (2) is the expectation value of the kinetic energy of the nuclear motion and the second term, which we will call $\left\langle Y^{\prime}\right\rangle$, is the magnetic and retardation interaction between the muon and the nucleus. All parts which are dependent upon the nuclear spin have been removed because the spin-spin interaction will be very small even in muonic atoms. This procedure is, of course, completely safe for all nuclei with spin 0. Equation (2) can be expressed in terms of the total energy of the bound muon, the nuclear potential, and the large 
and small components ( $g$ and $f$, respectively) of the wave function of the bound muon:

$$
\left\langle p^{2} / 2 M\right\rangle=\left(2 M c^{2}\right)^{-1} \int_{0}^{\infty}\left(c^{2} p_{0}^{2}-m^{2} c^{4}\right)\left(f^{2}+g^{2}\right) r^{2} d r
$$

with $c p_{0}=W+Z e^{2} / \overrightarrow{\mathrm{r}}$ and $\int_{0}^{\infty}\left(f^{2}+g^{2}\right) r^{2} d r=1$, and

$$
\langle Y\rangle=-\left(Z e^{2} / M c^{2}\right)\left\{\int_{0}^{\infty}\left\{\left(c p_{0}+m c^{2}\right) f^{2}+\left(c p_{0}-m c^{2}\right) g^{2}\right] r d r-\hbar \kappa c \int_{0}^{\infty} f g d r\right\}
$$

with the radial quantum number $k=j+\frac{1}{2}$.

The sum of both terms can be expressed by

$$
E_{1}=\left\langle\frac{p^{2}}{2 M}\right\rangle+\langle Y\rangle=Z e^{2} \frac{m}{M} \int_{0}^{\infty}\left(g^{2}-f^{2}\right) r d r-\frac{Z^{2} c^{4}}{2 M c^{2}}\left\langle r^{-2}\right\rangle+\frac{Z e^{2} \hbar k c}{M c^{2}} \int_{0}^{\infty} f g d r+\frac{W^{2}-m^{2} c^{4}}{2 M c^{2}} \text {. }
$$

Going to the limit of small $Z$ and the case of a pure Coulomb potential, the sum reduces to the expression

$$
E_{1} \approx-\left(W^{2}-m^{2} c^{4}\right) / 2 M c^{2} \text {. }
$$

This expression can be written for small binding energies as

$$
E_{1} \approx-\frac{m}{M}\left(W-m c^{2}\right)-\frac{m}{M}\left(\frac{Z \alpha}{2 n}\right)^{2}\left|W-m c^{2}\right|
$$

The first part in this equation is the reduced-mass correction in its simplest version, and the second part is identical with Eq. (1). Of course, this procedure is correct only in the case where the mean radius of the muonic wave function is large in comparison to the nuclear dimensions and where the mass of the nucleus is large in comparison to the mass of the muon. Thus, for low $-Z$ muonic atoms at least, Eq. (4) has to be used instead of Eq. (5).

To assess how reliable the various approximations are for the inner orbitals of a heavy muonic atom like lead we list the various approximations and calculations for comparison in Table I. Column 1 gives the reduced-mass correction in its simplest version by multiplying the energy eigenvalue by $m / M$. Columns 2 and 3 give the results according to Eqs. (5) and (4), respectively. To give an even

\begin{tabular}{|c|c|c|c|c|c|c|c|c|c|c|}
\hline \multirow[t]{2}{*}{ level } & \multirow{2}{*}{$\begin{array}{l}\quad 1 \\
\text { reduced } \\
\text { mass } \\
\text { correction } \\
\qquad\left|E_{i}\right| \cdot \frac{m}{M}\end{array}$} & \multirow{2}{*}{$\begin{array}{l}\quad 2 \\
\text { calculation } \\
\text { using Eq. (5) }\end{array}$} & \multirow{2}{*}{$\begin{array}{l}\quad 3 \\
\text { calculation } \\
\text { using Eq. (4) }\end{array}$} & \multicolumn{4}{|c|}{${ }^{4}$} & \multirow{2}{*}{$\begin{array}{l}\quad 5 \\
\text { reduced mass } \\
\text { correction } \\
\text { using the } \\
\text { reduced mass } \\
\text { in the nume- } \\
\text { rical integr. }\end{array}$} & \multirow{2}{*}{$\begin{array}{l}\quad 6 \\
\text { resulting } \\
\text { relativistic } \\
\text { reduced mass } \\
\text { correction }\end{array}$} & \multirow{2}{*}{$\begin{array}{c}7 \\
\text { approximation } \\
\text { acc. Eq. (7) }\end{array}$} \\
\hline & & & & $\begin{array}{l}\text { reduced } \\
\text { mass } \\
\text { correction } \\
\text { part }\end{array}$ & $\begin{array}{l}\text { magnetic } \\
\text { inter- } \\
\text { action }\end{array}$ & $\begin{array}{l}\text { retar- } \\
\text { dation }\end{array}$ & sum & & & \\
\hline Is $1 / 2$ & 5761 & 5245 & 5477 & 2998 & -923 & 343 & 2418 & 2780 & -362 & -582 \\
\hline $2 s_{1 / 2}$ & 1960 & 1916 & 1927 & 1574 & -363 & 75 & 1287 & 1387 & -100 & -117 \\
\hline $2 \mathrm{p}_{1 / 2}$ & 2619 & 2560 & 2561 & 2575 & -693 & 185 & 2067 & 2195 & -128 & \multirow[t]{2}{*}{-95} \\
\hline $2 \mathrm{p}_{3 / 2}$ & 2519 & 2463 & 2465 & 2391 & -452 & 211 & 2150 & 2224 & --74 & \\
\hline $3 s_{1 / 2}$ & 962 & 952 & 954 & 857 & -152 & 27 & 731 & 767 & -36 & -38 \\
\hline $3 p_{1 / 2}$ & 1165 & 1154 & 1154 & 1176 & -238 & 51 & 989 & 1028 & -39 & \multirow{2}{*}{-24} \\
\hline $3 p_{3 / 2}$ & 1140 & 1128 & 1129 & 1132 & -178 & 63 & 1017 & 1038 & -21 & \\
\hline $3 \mathrm{~d}_{3 / 2}$ & 1184 & 1172 & 1172 & 1272 & -171 & 61 & 1162 & 1176 & -13.5 & \multirow[t]{2}{*}{-11.8} \\
\hline${ }^{38} 5 / 2$ & 1161 & 1749 & 1150 & 1202 & -113 & 56 & 1145 & 1157 & $-12 \cdot 0$ & \\
\hline $4 f_{5 / 2}$ & 656 & 652 & 652 & 679 & -45 & 17 & 652 & 656 & -3.7 & -3.6 \\
\hline $5 g_{7 / 2}$ & 417 & 416 & 416 & 425 & -17 & 7 & 415 & 417 & -1.5 & -1.5 \\
\hline
\end{tabular}
better approximation for the effect of the extended nucleus on the inner orbitals in high- $Z$ muonic atoms we did not only use the relativistic wave functions calculated in the potential of the extended

TABLE I. Effect of the nuclear motion on various inner orbitals in muonic lead using the different approximations described in the text. Energies are in $\mathrm{eV}$. 
nucleus, but have also introduced, instead of the interaction parameter $Z e^{2}$, the nuclear potential itself, $r V(r)$. So Eq. (3) can be written as

$$
E_{1}=\frac{m}{M} \int_{0}^{\infty} V(r)\left(g^{2}-f^{2}\right) r^{2} d r-\frac{1}{2 M c^{2}} \int_{0}^{\infty} V(r)^{2}\left(f^{2}+g^{2}\right) r^{2} d r+\frac{\hbar \kappa c}{M c^{2}} \int_{0}^{\infty} V(r) f g r d r+\frac{W^{2}-m^{2} c^{2}}{2 M c^{2}} .
$$

In column 4 of Table I we list the results calculated with this equation. In this row we also list separately the expectation values $\left\langle p^{2} / 2 M\right\rangle$, the reduced-mass correction part, and $\langle Y\rangle$, the effect of the magnetic and retardation interaction between the muon and the nucleus. The magnetic interaction increases the binding energy, whereas the retardation effect decreases the binding of the muon.

This value in column 4 has to be compared with the calculation usually used where the reduced-mass correction is directly taken into account in the numerical integration of the Dirac equation. The difference between such a calculation using the reduced mass and the rest mass of the muon is given in column 5. In such a calculation, all influences due to the extended nucleus are already taken care of in an accurate way so that the difference between columns 4 and 5 , which we present in column 6 , gives the additional relativistic reduced-mass correction which we were looking for. A comparison of 2 and 4 also shows that the calculation of the relativistic reduced-mass correction which was used so far is correct for a transition like $5 g \rightarrow 4 f$, but is not adequate for inner transitions.

The results in column 6 can be checked independently for high levels in the following way. Within the Pauli approximation, the Breit Hamiltonian for a nucleus with spin 0 and a bound fermion can be written as the sum of (a) the nonrelativistic Hamiltonian for both particles, (b) the mass velocity, the spin-orbit, and the Darwin terms for both particles, and (c) the magnetic and retardation correction. When the reduced mass of the system is introduced instead of $m$ and $M$, the Hamiltonian ${ }^{10}$ reads (for a point nucleus), within the zeroth and first power of $m / M$, as

$$
\begin{aligned}
& H=H_{a}+H_{b}, \quad H_{a}=\left[V(r)+\frac{p^{2}}{2 \mu}\right]-\frac{p^{4}}{8 \mu^{3} c^{2}}+\frac{1}{r^{3}} \frac{Z e^{2} \hbar}{\mu c}(\overrightarrow{\mathrm{r}} \times \overrightarrow{\mathrm{p}}) \cdot s-\frac{i Z e^{2} \hbar}{(2 \mu c)^{2}} \overrightarrow{\mathrm{p}} \cdot \operatorname{grad} \frac{1}{r}, \\
& H_{b}=\frac{1}{M c^{2}}\left[\frac{3}{8 m^{2}} p^{4}-\frac{Z e^{2}}{2 m} \frac{1}{r}\left(p^{2}+p_{r}{ }^{2}\right)+\frac{i Z e^{2} \hbar}{2 m} \overrightarrow{\mathrm{p}} \cdot \operatorname{grad} \frac{1}{r}\right],
\end{aligned}
$$

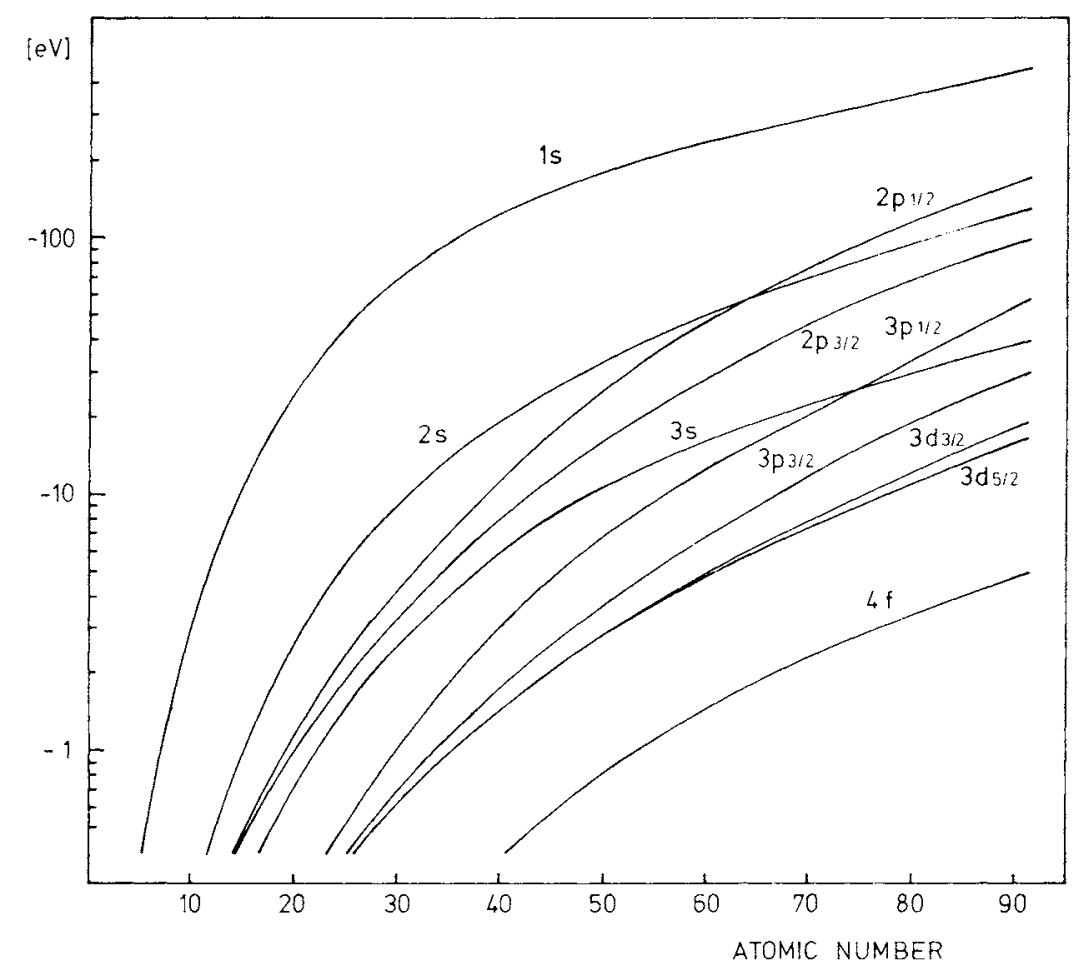

FIG. 1. Relativistic reduced-mass correction (in $\mathrm{eV}$ ) for the inner orbitals of muonic atoms as functions of the atomic number. 
with $p_{r}^{2}=\left(1 / r^{2}\right) \vec{r} \cdot(\vec{r} \cdot \vec{p}) \vec{p}$.

The first part, $H_{a}$, is the Pauli approximation to the Dirac Hamiltonian with the reduced mass. This is usually solved exactly by numerical integration of the Dirac equation itself. The expectation value of the second part, $H_{b}$, leads to the additional relativistic reduced-mass correction. Because this Hamiltonian is an approximation within nonrelativistic basis functions, the expectation value of $H_{b}$ can be calculated for $s, p, d, \ldots$ states only. These values, including the effect of the extended nucleus, are given in column 7 of Table I, and they show indeed a very good agreement for the high levels.

This result is very important because of the following two consequences. First, it shows that the theoretical calculations for the muonic transitions between states with large $n$ and $l$ values, which are used in the experiment to investigate the vacuum polarization in higher orders as well as shielding effects, ${ }^{11,12}$ are not affected by this contribution.

Secondly, the additional corrections for the innermost shells in heavy muonic atoms are as high as $1 \mathrm{keV}$ and the sum of all additional effects as high as $0.5 \mathrm{keV}$. In view of the high-accuracy measurements of the transitions in muonic atoms, the exact knowledge of these corrections becomes most important. Recent experimental determinations of the nuclear polarization ${ }^{13}$ lie systematically above the theoretical values of Chen and Skarohamar. ${ }^{6}$ Although these corrections are too small to explain these differences, they go in the right direction. In Fig. 1 we give the corrections of the relativistic reduced-mass effect for the inner levels as functions of $Z$.
I would like to thank Dr. Waber, Dr. Kankeleit, and Dr. Vogel for many interesting discussions.

${ }^{*}$ Research supported by a grant from the U.S. Atomic Energy Commission.

$\dagger$ Present address: Gesellschaft fur Schwerionenforschung mbH, Darmstadt, Postfach 541, W. Germany.

${ }^{1} \mathrm{~S}$. Devons and J. Duderoth, Advan. Nucl. Phys. $\underline{2}$, 295 (1969); C. S. Wu and L. Wilets, Annu. Rev. Nucl. Sci. 19, 527 (1969); K. W. Ford and J. G. Wills, Phys. Rev. 185,1429 (1969), and references therein.

${ }^{2}$ E. A. Uehling, Phys. Rev. 48, 55 (1935); J. Schwinger, Phys. Rev.75, 651 (1969).

${ }^{3}$ R. J. McKee, Phys. Rev. 180, 1139 (1969).

${ }^{4}$ B. Fricke, Z. Phys. 218, $\overline{495}$ (1969).

${ }^{5}$ R. C. Barrett, J. S. Brodsky, G. W. Erickson, and M. H. Goldhaber, Phys. Rev. 166, 1589 (1968); R. C. Barrett, Phys. Lett. 28B, 93 (1968).

${ }^{6}$ M. Y. Chen, Phys. Rev. C 1, 1167 (1970), and references therein; H. F. Skarohamar, Nucl. Phys. A151, 154 (1970); K. Tanabe, Phys. Rev. A 3 , 1282 (1971).

${ }^{7}$ B. Fricke, Lett. Nuovo Cimento 2, 859 (1969).

${ }^{8} \mathrm{~K}$. Bechert and J. Meixner, Ann. Phys. (Leipzig) 22, 525 (1935).

${ }^{9}$ G. Breit and G. E. Brown, Phys. Rev. 74, 1278 (1948).

${ }^{10} \mathrm{H}$. A. Bethe and E. E. Salpeter, in Handbuch der Physik, edited by S. Flügge (Springer, Berlin, 1957), Vol. 35, Chap. 42.

${ }^{11}$ M. S. Dixit, H. L. Anderson, C. K. Hargrove, R. J. McKee, D. Kessler, H. Mes, and A. C. Thompson, Phys. Rev. Lett. 27, 878 (1971); M. S. Dixit, Ph. D. thesis, University of Chicago, 1971 (unpublished).

${ }^{12}$ B. Fricke, J. T. Waber, and V. L. Telegdi, to be published.

${ }^{13} \mathrm{P}$. Martin, College of William and Mary Internal Report No. WM-38-72, 1972 (unpublished); D. A. Jenkins, R. J. Powers, P. Martin, H. G. Miller, and R. E. Welsh, Nuc1. Phys. A175, 73 (1971). 